\title{
Modified reperfusion in clinical lung transplantation: The results of 100 consecutive cases
}

\author{
Gabriel T. Schnickel, MD, a David J. Ross, MD, ${ }^{\text {b }}$ Ramin Beygui, MD, ${ }^{\text {a }}$ Ali Shefizadeh, BS, ${ }^{a}$ Hillel Laks, MD, \\ Rajan Saggar, MD, Joseph P. Lynch III, MD, and Abbas Ardehali, MD
}

From the Division of Cardiothoracic Surgery, Department of Surgery, ${ }^{\mathrm{a}}$ and Division of Pulmonary Medicine, Critical Care and Hospitalists, ${ }^{\mathrm{b}}$ David Geffen School of Medicine, University of California, Los Angeles, Calif.

Read at the Eighty-fifth Annual Meeting of The American Association for Thoracic Surgery, San Francisco, Calif, April 10-13, 2005.

Received for publication April 21, 2005; revisions received Aug 15, 2005; accepted for publication Aug 30, 2005.

Address for reprints: Gabriel T. Schnickel, MD, Division of Cardiothoracic Surgery, 62182 CHS, UCLA Medical Center, 10833 Le Conte Ave, Los Angeles, CA 90095 (E-mail: gschnick@ucla.edu).

J Thorac Cardiovasc Surg 2006;131:218-23

$0022-5223 / \$ 32.00$

Copyright $\odot 2006$ by The American Association for Thoracic Surgery

doi:10.1016/j.jtcvs.2005.08.045
Objective: Severe primary graft dysfunction occurs in $10 \%$ to $20 \%$ of lung transplant recipients and is the leading cause of early death after lung transplantation. We hypothesized that altering the content of the initial reperfusate and maintaining a low reperfusion pressure after surgical implantation would lead to a low incidence of primary graft dysfunction.

Methods: We analyzed the records of all patients who underwent lung transplantation at our institution from March 1, 2000, to August 30, 2004. The modified reperfusion technique involved the insertion of a catheter into the main or individual pulmonary artery after implantation. The recipient blood was depleted of leukocytes; supplemented with nitroglycerin; adjusted for $\mathrm{pH}$ and calcium level; enriched with aspartate, glutamate, and dextrose; and then administered into the pulmonary arteries of the newly transplanted lung(s) for the first 10 minutes of reperfusion. Severe primary graft dysfunction was defined as a $\mathrm{PaO}_{2} /$ inspired oxygen fraction of less than 150 with diffuse infiltrate on the radiograph in absence of other causes.

Results: During this interval, 100 patients underwent lung transplantation with the modified reperfusion technique. Forty-two patients underwent single-lung transplantation, of which 5 patients required cardiopulmonary bypass for the procedure. Fifty-eight patients underwent double-lung transplantation; all double-lung transplantation procedures were performed with patients on cardiopulmonary bypass. There were no technical complications associated with the modified reperfusion. The mean $\mathrm{PaO}_{2} /$ inspired oxygen fraction at 6 hours in this cohort was $252 \pm 123 \mathrm{~mm} \mathrm{Hg}$. The median number of days on the ventilator was 2. More importantly, the incidence of severe primary graft dysfunction in this cohort was $2.0 \%$. The early survival (30-day or in-hospital mortality) of this group of patients was $97 \%$.

Conclusions: The technique of modified reperfusion in human lung transplantation is associated with a low incidence of severe primary graft dysfunction and favorable short-term outcomes.

$\mathrm{L}$ ung transplantation is a well-established treatment option for patients with end-stage lung diseases. Approximately 1500 lung transplantations are performed annually, and more than 17,000 have been performed worldwide though 2004. ${ }^{1}$ Despite this breadth of experience and improved donor management and preservation techniques, ischemia-reperfusion injury or primary graft dysfunction (PGD) remains a significant cause of early morbidity and mortality after lung transplantation. Although PGD refers to a spectrum of lung allograft injuries after transplantation, its most severe form is estimated to occur in $10 \%$ to $20 \%$ of lung transplant recipients with an associated mortality of $40 \%{ }^{2,3}$ The syndrome generally occurs within the first 72 hours after transplantation and is characterized by nonspecific alveolar damage, lung edema, poor compliance, and hypoxemia. The treatment for PGD remains primarily supportive, and in addition to the high mortality associated with this phenomenon, 


\section{Abbreviations and Acronyms \\ $\mathrm{CPB}=$ cardiopulmonary bypass \\ $\mathrm{FIO}_{2}=$ inspired oxygen fraction \\ ICU $=$ intensive care unit \\ $\mathrm{PGD}=$ primary graft dysfunction}

the survivors have a prolonged recovery and are frequently left with residual impaired lung function. ${ }^{3}$ Severe PGD can also be associated with an increased risk of acute rejection and late bronchiolitis obliterans syndrome. ${ }^{4}$

Recipient leukocytes are known to play an integral role in the development of PGD in lung transplantation. Experimental studies have shown that neutrophils and T lymphocytes infiltrate the lung allografts and contribute to graft injury through a variety of mechanisms, including the release of toxic oxygen metabolites, inflammatory cytokines, and other mediators. ${ }^{5,6}$ In addition to recipient leukocytes, the noncellular composition of the reperfusate also contributes to the process of ischemia-reperfusion injury by exacerbating cellular edema and death. ${ }^{7}$ Modification of the initial reperfusate after regional myocardial ischemia has been associated with improved functional recovery. ${ }^{7}$ Moreover, application of this technique in experimental models of lung transplantation has resulted in improved allograft function and oxygenation. ${ }^{8,9}$ We have previously reported on our experience with modified reperfusion in a cohort of 23 patients. ${ }^{10}$ The purpose of this study was to assess the shortand medium-term outcome of modified reperfusion in a group of 100 consecutive lung transplant recipients.

\section{Materials and Methods Study Population}

We analyzed the records of all patients who underwent lung transplantation at our institution from March 1, 2000, to August 30, 2004. During this interval, 100 patients had received modified reperfusion with their lung transplantation, and they are the subjects of this study. One patient during this time period did not receive modified reperfusion because of the decision of the surgeon. The first 12 lung transplant recipients included in this cohort were enrolled in a previously reported study. ${ }^{10}$ This study was approved by the University of California, Los Angeles Institutional Review Board.

\section{Allograft Harvest and Implantation}

Donor lungs were harvested by using the previously described technique. ${ }^{11}$ Donor lung pulmonary artery beds were pretreated with $500 \mu \mathrm{g}$ of prostaglandin $\mathrm{E}_{1}$, followed by administration of 4 L of either modified Euro-Collins solution (the first 41 recipients; Baxter Healthcare Corp, Deerfield, Ill) or low-potassium dextran solution (the last 59 recipients; Perfadex; Vitrolife, Uppsala, Sweden) under a perfusion pressure less than $20 \mathrm{~mm} \mathrm{Hg}$. All allografts were procured in inflation with a tidal volume of approximately $10 \mathrm{~mL} / \mathrm{kg}$ and with a inspired oxygen fraction $\left(\mathrm{FIO}_{2}\right)$ of 1.0. Meth- ylprednisolone $(7 \mathrm{mg} / \mathrm{kg}$ ) was given to all recipients before surgery. Allograft implantation was performed according to a previously described technique. ${ }^{12}$ All double lung transplantations were performed on cardiopulmonary bypass (CPB). Allograft ischemia time for this group of patients refers to the time until both lung allografts were reperfused. Aprotinin is used in all patients who undergo lung transplantation on CPB.

\section{Modified Reperfusion}

After implantation of a right or left single-lung allograft (off CPB), a cannula was inserted into the right atrium or descending thoracic aorta, respectively. ${ }^{10}$ The recipient blood was removed, depleted of leukocytes via leukofilter (LeukoGuard BC1B for the first 6 recipients and then LeukoGuard LG6B; Pall Biomedical Products, East Hills, NY), and then mixed with the modified reperfusion solution in a 4:1 ratio via a roller pump. The efficacy of leukofilters in removal of leukocytes has previously been demonstrated in both experimental and clinical studies. ${ }^{13,14}$ The precise content of the modified reperfusion solution has previously been described. ${ }^{10}$ The modified reperfusate was then administered through a cannula that had been inserted into the clamped pulmonary artery via the suture line. The modified reperfusion pressure was continuously monitored and maintained at a pressure less than $20 \mathrm{~mm} \mathrm{Hg}$. The flow varied from 200 to $500 \mathrm{~mL} / \mathrm{min}$ per lung allograft. During reperfusion, patients were ventilated in a pressure-controlled mode with tidal volume of approximately $8 \mathrm{~mL} / \mathrm{kg}$ on room air and with a positive end-expiratory pressure of $5 \mathrm{~mm} \mathrm{Hg}$. The effluent during the first 3 minutes of reperfusion was collected by a cell-saving device (in patients undergoing transplantation without CPB). After the first 3 minutes of reperfusion and after adequate deairing of the allograft, the left atrial clamp was removed to allow return of the leukocyte-depleted blood into the recipient's left atrium. After 3 minutes, the modified reperfusion solution was switched to leukocyte-depleted blood, which was continued for an additional 7 minutes. Activated clotting time was maintained over 200 seconds during the reperfusion phase.

In patients undergoing single-lung transplantation on $\mathrm{CPB}$, the pump blood was used to reperfuse the allograft after leukofiltration and mixing with the modified reperfusate solution. In patients undergoing double-lung transplantation on $\mathrm{CPB}$, the modified reperfusate was administered into the main pulmonary artery in a similar fashion at a pressure less than $20 \mathrm{~mm} \mathrm{Hg}$. After 10 minutes of modified reperfusion, the removal of the pulmonary artery clamp or weaning off $\mathrm{CPB}$ was performed according to usual protocol.

\section{Postoperative Care}

All patients were mechanically ventilated in a pressure-controlled mode with settings adjusted to maintain $\mathrm{PaO}_{2}$ greater than $80 \mathrm{~mm}$ $\mathrm{Hg}$. Positive end-expiratory pressures of 3 to $10 \mathrm{~mm} \mathrm{Hg}$ were used to optimize oxygenation and hemodynamics. The immunosuppression regimen initially consisted of induction with equine antithymocyte globulin ( $\mathrm{n}=5$; ATGam; Pharmacia, Peapack, NJ), followed by cyclosporine, azathioprine, and prednisone. The regimen was changed in January 2001 and has subsequently consisted of induction therapy with rabbit anti-thymocyte globulin (Thymoglobulin; SangStat, Fremont, Calif), followed by tacrolimus, mycophenolate mofetil, and prednisone. The antithymocyte globulin was administered after surgery. Routine transbronchial lung biop- 
sies were performed per protocol at postoperative weeks $4,8,12$, 24 , and 52. Clinically based procedures were based on decrements in spirometric function or radiographic infiltrate or on decreased pulse oximetry.

\section{Definition of Terms}

Severe PGD was defined as $\mathrm{PaO}_{2} / \mathrm{FIO}_{2}$ less than 150 at 48 hours after transplantation with radiographic evidence of pulmonary edema in mechanically ventilated patients, in the absence of other potential causes. The $\mathrm{PaO}_{2}$ value and chest radiograph closest to 6 and 48 hours after arrival in the intensive care unit (ICU) were used for data collection for this study. Acute cellular rejection was diagnosed by transbronchial lung biopsy and graded according to the International Society for Heart and Lung Transplantation guidelines: grade 0 , no rejection; grade $\mathrm{A} 1$, minimal; grade $\mathrm{A} 2$, mild; grade A3, moderate; and grade A4, severe. Early deaths were defined as deaths within 30 days of the operation or during the same hospitalization period. All data measurements are presented as the mean $\pm \mathrm{SD}$, unless otherwise specified.

\section{Results}

\section{Perioperative Characteristics}

The recipient diagnoses were obstructive lung disease $(\mathrm{n}=$ $45)$, restrictive lung disease $(\mathrm{n}=42)$, pulmonary vascular disease $(n=5)$, and septic lung disease $(n=8)$. The mean age of recipients was $57 \pm 9$ years. The most recently available recipient mean pulmonary artery pressure preceding the transplantation procedure was $38 \pm 8 \mathrm{~mm} \mathrm{Hg}$. During this study period, 69 recipients received lung allografts from donors who met extended donor criteria. The lungs were classified as extended donor lungs for the presence of radiographic infiltrate in the donor lung $(n=61)$, donor age older than 55 years $(\mathrm{n}=8)$, donor $\mathrm{PaO}_{2} / \mathrm{FIO}_{2}$ less than 300, an $\mathrm{FiO}_{2}$ of $1.0(\mathrm{n}=5)$, the presence of purulent secretions $(n=28)$, and a smoking history greater than 20 pack-years $(\mathrm{n}=7)$. Several donors met multiple criteria.

Forty-two patients underwent single-lung transplantation, of which 5 patients required $\mathrm{CPB}$ for the procedure. Fifty-eight patients underwent double-lung transplantation; all double-lung transplantations were performed on CPB, with a mean bypass time of $191 \pm 35$ minutes. During this interval, 21 patients underwent a concomitant cardiac procedure at the time of transplantation. These operations included mitral valve repair $(n=1)$, tricuspid valve repair $(n=4)$, tricuspid and pulmonic valve repair $(\mathrm{n}=1)$, coronary artery bypass graft $(n=2)$, and patent foramen ovale closure $(n=13)$. The mean allograft ischemia time for all transplantations was $310 \pm 63$ minutes. There were no pulmonary artery injuries, and there was no postoperative bleeding related to the modified reperfusion technique.

\section{Postoperative Outcome}

The early postoperative characteristics of this cohort of patients are shown in Table 1. There were no intraoperative deaths. The mean $\mathrm{PaO}_{2} / \mathrm{FIO}_{2}$ at 6 hours after transplantation
TABLE 1. Early postoperative characteristics

\begin{tabular}{lc}
\hline Variable & Data \\
\hline $\mathrm{PaO}_{2} / \mathrm{FlO}_{2}$ at $6 \mathrm{~h}$ & $252 \pm 123$ \\
$\mathrm{PaO}_{2} / \mathrm{FlO}_{2}$ at $48 \mathrm{~h}$ & $301 \pm 80$ \\
$\mathrm{Days}$ on ventilator, median (range) & $2(1-202)$ \\
Days in ICU, median (range) & $4(2-202)$ \\
Length of stay, d, median (range) & $14(6-277)$ \\
Incidence of severe PGD (at $48 \mathrm{~h})$ & $2.0 \%$ \\
Incidence of acute rejection & $0.23 \pm 0.47$ \\
Early survival & $97 \%$ \\
One-year survival & $91 \%$ \\
\hline
\end{tabular}

$\mathrm{FlO}_{2}$, Fraction of inspired oxygen; $I C U$, intensive care unit; $P G D$, primary graft dysfunction. Data are mean $\pm S D$ unless otherwise noted.

was $252 \pm 123 \mathrm{~mm} \mathrm{Hg}$. At 6 hours after ICU arrival, patients had $\mathrm{PaO}_{2} / \mathrm{FIO}_{2}$ ratios of less than 150 . Thirteen of these patients received donor lungs with infiltrates (due to atelectasis, contusion, or pneumonia), and postoperative chest radiographs (on arrival in the ICU) demonstrated the persistence of similar infiltrates. One patient had hyperacute rejection, as demonstrated by donor-specific positive crossmatching and consistent histologic findings. Another patient had a $\mathrm{PaO}_{2} / \mathrm{FIO}_{2}$ ratio less than 150 with a clear radiograph. Finally, 3 patients had a $\mathrm{PaO}_{2} / \mathrm{FIO}_{2}$ ratio less than 150 with diffuse radiographic infiltrate at 6 hours. A factor that may have contributed to the hypoxemia in this cohort is the relatively high rate of CPB use (63 of 100) and the associated early lung edema.

The mean $\mathrm{PaO}_{2} / \mathrm{FIO}_{2}$ at 48 hours after transplantation was $301 \pm 80 \mathrm{~mm} \mathrm{Hg}(\mathrm{n}=50$ for the group that was mechanically ventilated at 48 hours). At 48 hours after ICU arrival, 3 patients had $\mathrm{PaO}_{2} / \mathrm{FIO}_{2}$ ratios less than 150 . One patient had hyperacute rejection, as noted previously. Two patients had a diagnosis of severe PGD.

In this group of 100 patients, the median number of days on the ventilator was 2 , the median number of days in the ICU was 4, and the median length of hospital stay was 14 days. In a previous report, acute renal failure developed in 2 patients as a result of hemolysis. ${ }^{10}$ This was possibly due to high flows through the leukocyte filter. The filters were subsequently changed to high-flow leukocyte filters, with no subsequent episodes of hemolysis.

Two (2.0\%) patients from this cohort met the criteria for severe PGD at 48 hours. Both of these patients had the diagnosis of pulmonary arterial hypertension and had undergone double-lung transplantation. One of these patients had had a prior sternotomy and intracardiac repair; she required femoral artery and vein cannulation and prolonged CPB for lung transplantation and redo tricuspid valve repair. This patient had a single episode of acute rejection during the first posttransplantation year. The second patient had also required tricuspid valve repair and double lung transplantation. She has had multiple episodes of A2 or 
greater acute rejection since transplantation and was diagnosed with bronchiolitis obliterans syndrome (stage 3) at 2 years after transplantation.

The incidence of airway complications requiring intervention during the first posttransplantation year was $0 \%$. The incidence of acute rejection (grade A2 or higher) during the first year after transplantation was $0.23 \pm 0.47$ episodes per patient-year. There were no significant differences in any of the end points when they were stratified by preservation solution (data not shown). There were 3 early deaths in this cohort due to multisystem organ failure (postoperative day 17), sepsis (postoperative day 202), and drug overdose in an outpatient (postoperative day 24). The early survival of this group of patients was $97 \%$, with a 1-year actuarial survival of $91 \%$.

\section{Discussion}

The findings of this study demonstrate that modifying the initial reperfusate by leukodepletion and altering the composition of the solution can be performed safely in human lung transplantation. Moreover, this technique is associated with a low incidence of severe PGD and with favorable early and 1-year outcomes.

PGD in lung transplantation is a complex multifactorial process that involves participation of innate and adaptive immune systems, complement cascade, reactive oxygen species, and coagulation factors. Given the complexity of this process, it is not surprising that therapies directed at single pathogenic factors might prove ineffective. The described technique allows control of both the content and the conditions of the initial reperfusion after surgical implantation. The importance of controlling the content of the reperfusate after a period of ischemia has been substantiated in several experimental and clinical studies. ${ }^{7,9,15,16}$ Recipient leukocytes and, specifically, neutrophils and $\mathrm{T}$ lymphocytes can mediate ischemia reperfusion injury through a variety of mechanisms, such as release of toxic oxygen metabolites, elaboration of elastases and proteases, production of inflammatory cytokines, and neutrophil aggregation, thus causing plugging of capillaries and no-reflow phenomena., ${ }^{5,6,17-19}$ Removal of leukocytes from the reperfusate has been shown to improve cardiac allograft histologic characteristics in a clinical study. ${ }^{20}$ Thus, it is possible that leukofiltration may account for at least part of the beneficial effects of modified reperfusion in this study. In addition to leukocytes, the acellular composition of the reperfusate is also important in mediating the severity of the graft injury. ${ }^{7}$ The composition of the reperfusate in this study was based on theoretical grounds, with the goals of neutralizing intracellular and extracellular acidosis, minimizing tissue edema, enriching the cellular energy stores by providing the substrates, minimizing calcium-induced cellular damage, and ensuring homogenous delivery.
In addition to controlling the content of the reperfusate, the condition of the reperfusion is also critical in determining the severity of graft injury. Experimental studies have demonstrated that maintaining the reperfusion pressure at less than $20 \mathrm{~mm} \mathrm{Hg}$ can reduce pulmonary edema and improve allograft function. ${ }^{21}$ An integral part of the described reperfusion technique is to control the reperfusion pressure at less than $20 \mathrm{~mm} \mathrm{Hg}$ for the first 10 minutes of reperfusion. On the basis of this study, we cannot decipher which component of modified reperfusion technique (leukofiltration vs modification of the acellular components vs controlling the pressure) is the dominant factor in mediating the beneficial effects of this technique.

A limitation of this study is the lack of a control group; the absence of a contemporaneous cohort does not allow direct comparison of this approach. The technique of modified reperfusion is not without its limitations. To perform modified reperfusion, as described, requires an additional 10 minutes of operative time and increased CPB time for transplantations performed with patients on bypass. For singlelung transplantations, this technique requires an additional cannulation site and the increased risk with which it is associated. There is also an increased risk of bleeding secondary to the anticoagulation the technique requires and the risk of thrombocytopenia, although our early experience had not demonstrated this to be a problem. ${ }^{10}$ Because this technique largely relies on the removal of leukocytes from the reperfused blood, there may also be a heightened risk of infection. Moreover, the use of modified reperfusion does require additional costs. Finally, this technique does not eliminate the risk of PGD; patients with pulmonary arterial hypertension who are at high risk of PGD may experience this complication despite modified reperfusion ( 2 of 5 patient in this cohort).

Given the scarcity of "ideal" donor lungs, many transplantation centers have expanded the criteria for donor lung acceptance, with favorable short- and medium-term outcomes. ${ }^{22,23}$ A major concern regarding the use of "nonideal" donor lungs is the risk of severe PGD. Strategies such as modified reperfusion that may decrease the risk of PGD may allow use of nonideal lungs and expansion of the donor pool.

PGD is the result of the cumulative effects of allograft injury that occur secondary to donor brain death, organ harvest, cold preservation, and reperfusion. Effective control of allograft function must address each of these distinct processes. The technique of modified reperfusion focuses predominantly on the issues of reperfusion. A comprehensive approach to the management of PGD must include attention to donor management, organ harvest, and preservation, in addition to reperfusion. Furthermore, this technique is only a vehicle for controlling the reperfusion process. With greater understanding of 
the pathophysiology of reperfusion injury, the content of the reperfusate is likely to, and should, change.

\section{References}

1. Trulock EP, Edwards LB, Taylor DO, Boucek MM, Keck BM, Hertz MI. The registry of the International Society for Heart and Lung Transplantation: twenty-first official adult lung and heart-lung transplant report-2004. J Heart Lung Transplant. 2004;23:804-15.

2. King RC, Binns OA, Rodriguez F, Kanithanon RC, Daniel TM, Spotnitz WD, et al. Reperfusion injury significantly impacts clinical outcome after pulmonary transplantation. Ann Thorac Surg. 2000;69: 1681-5.

3. Christie JD, Bavaria JE, Palevsky HI, et al. Primary graft failure following lung transplantation. Chest. 1998;114;51-60.

4. Fiser SM, Tribble CG, Long SM, Kaza AK, Kern JA, Jones DR, et al. Ischemia-reperfusion injury after lung transplantation increases risk of late bronchiolitis obliterans syndrome. Ann Thorac Surg. 2002;73: 1041-7.

5. Carden DL, Xiao F, Moak C, Willis BH, Robinson-Jackson S, Alexander S. Neutrophil elastase promotes lung microvascular injury and proteolysis of endothelial cadherins. Am J Physiol Heart Circ Physiol. 1998;275:385-92.

6. Panes J, Perry M, Granger DN. Leukocyte endothelial cell adhesion: avenues for therapeutic intervention. Br J Pharmacol. 1999;126:537-50.

7. Allen BS, Okamoto F, Buckberg GD, Bugyi H, Leaf J. Studies of controlled reperfusion after ischemia. XV. Immediate functional recovery after 6 hours of regional ischemia by careful control of conditions of reperfusion and composition of the reperfusate. $J$ Thorac Cardiovasc Surg. 1986;92:621-35.

8. Halldorsson AO, Kronen M, Allen BS, Rahman S, Wang T, Layland $\mathrm{M}$, et al. Controlled reperfusion prevents pulmonary injury after 24 hours of lung preservation. Ann Thorac Surg. 1998;66:877-84.

9. Halldorsson AO, Kronon M, Allen BS, Rahman S, Wang T, Layland $\mathrm{M}$, et al. Controlled reperfusion after lung ischemia: implications for improved function after lung transplantation. J Thorac Cardiovasc Surg. 1998;115:415-25.

10. Ardehali, Laks H, Russell H, Levine M, Shpiner R, Lackey S, et al. Modified reperfusion and ischemia-reperfusion injury in human lung transplantation. J Thorac Cardiovasc Surg. 2003;126:1929-34.

11. Sundaresan S, Trachiotis GD, Aoe M, Patterson GA, Copper JD. Donor lung procurement: assessment and operative technique. Ann Thorac Surg. 1990;49:785-91.

12. Pasque MK, Cooper JD, Kaiser LR, Haydock DA, Traintafillou A, Trulock EP. Improved technique for bilateral lung transplantation: rationale and initial clinical experience. Ann Thorac Surg. 1998;66: 877-85.

13. Heggie AJ, Corder JS, Crichton PR, Hesford JW, Bingham H, Jeffries S. Clinical evaluation of the new Pall leucocyte-depleting blood cardioplegia filter (BC1). Perfusion. 1998;13:17-25.

14. Clavien PA, Harvey PR, Sanabria JR, Cywes R, Levy GA, Strasberg SM. Lymphocyte adherence in the reperfused rat liver: mechanisms and effects. Hepatology. 1993;17:131-42.

15. Lu Y, Hellewell P, Evans T. Ischemia-reperfusion lung injury: contribution of ischemia, neutrophils and hydrostatic pressure. Am J Physiol. 1997;273(Suppl):L46-54.

16. Breda MA, Hall TS, Stuart RS, Baumgartner WA, Borkon AM, Brawn $\mathrm{JD}$, et al. Twenty-four hour lung preservation by hypothermia and leukocyte depletion. J Heart Transplant. 1985;4:325-9.

17. Rezkalla SH, Kloner RA. No-reflow phenomenon. Circulation. 2002; 105:656-62.

18. de Perrot M, Young K, Imai Y, Fischer S, Liu M, Waddell TK, et al. Recipient $\mathrm{T}$ cells mediate reperfusion injury after lung transplantation in the rat. J Immunol. 2003;171:4995-5002.

19. Burne MJ, Daniels F, El Ghandour A, Mauiyyedi S, Colvin RB, O'Donnell MP, et al. Identification of the CD4(+) T cell as a major pathogenic factor in ischemic acute renal failure. J Clin Invest. 2001; 108:1283-90.

20. Pearl JM, Drinkwater DC, Laks H, Stein DG, Capouya ER, Bhuta S. Leukocyte-depleted reperfusion of transplanted human hearts prevents ultrastructural evidence of reperfusion injury. $J$ Surg Res. 1992;52:298-308.

21. Bhabra MS, Hopkinson DN, Shaw TE, Hooper TL. Critical importance of the first 10 minutes of lung graft reperfusion after hypothermic storage. Ann Thorac Surg. 1996;61:1631-5.

22. Meyers BF, Lynch J, Trulock EP, et al. Lung transplantation: a decade of experience. Ann Surg. 1999;230:362-70.

23. Bhorade SM, Vigneswaran W, McCabe MA, Garrity ER. Liberalization of donor criteria may expand the donor pool without adverse consequence in lung transplantation. J Heart Lung Transplant. 2000; 19:1199-204.

\section{Discussion}

Dr Wickii T. Vigneswaran (Maywood, Ill). I would like to congratulate Dr Schnickel and his colleagues for an elegant presentation of their report.

A severe form of pulmonary graft dysfunction remains a significant cause of morbidity and mortality following lung transplantation. Increasing knowledge of the mechanism of primary graft failure has led to improvements in donor management, organ preservation, and recipient management in the perioperative period. Further measures to reduce early graft dysfunction are important to improve outcomes. The authors have demonstrated that reperfusion of the lung allograft with a modified reperfusate following single or bilateral lung transplantation is feasible and safe.

However, I have some concerns about your conclusion that the technique of modified reperfusion is associated with a low incidence of severe pulmonary graft dysfunction and favorable shortterm outcomes. This is an uncontrolled retrospective review. Furthermore, the cohort of patients in this report is heterogeneous.

Two different techniques were used for preservation of the donor. Forty-two percent of the patients underwent a single-lung transplantation. In $63 \%$ of the patients, a CPB was used for the transplantation. In single-lung transplant, the remaining native lung can influence the $\mathrm{PaO}_{2} / \mathrm{FIO}_{2}$ ratio. The conduct of CPB during lung transplantation can modify the inflammatory response. I would like to ask you whether you used aprotinin, a potent anti-inflammatory agent, or leukocyte depletion of the prime or in the cardiopulmonary circuit during your operation. I would also like to know whether any subset of your patients received nitric oxide before reperfusion.

Dr Schnickel. Thank you for your comments.

Regarding aprotinin, yes, we do use aprotinin on those patients who undergo transplant on bypass. In regards to the nitric oxide, we currently use nitric oxide only when indicated for either elevated pulmonary artery pressures or for poor oxygenation. We don't routinely use it as part of our protocol.

Dr Vigneswaran. So there was a subset of patient who received nitric oxide but after the reperfusion of the graft?

Dr Schnickel. Correct.

Dr Vigneswaran. The incidence of primary graft dysfunction in the literature varies from single digits to over $30 \%$. One of the major setbacks of evaluating the results in the literature is the absence of uniformity in the definition of primary graft dysfunction. The most frequently used definition is $\mathrm{PaO}_{2} / \mathrm{FIO}_{2}$ ratio of less than 200 with diffuse infiltrates in the chest radiograph. The working group of the International Society for Heart and Lung Transplantation proposed a classification system to make it uniform, and the grade 3, which is to denote the severe form of pulmonary graft dysfunction, was defined as a $\mathrm{PaO}_{2} / \mathrm{FIO}_{2}$ ratio less than 200 with diffuse infiltrates in the chest radiograph. The 
definition used in your report is a ratio of less than 150. By setting the threshold high, the observed incidence of primary graft dysfunction can be lowered artificially. In your table, the $\mathrm{PaO}_{2} / \mathrm{FIO}_{2}$ ratio at 1 hour was $187 \pm 112.9$. I would like you to comment: is the incidence of primary graft dysfunction lower because of the definition you used and how you analyzed the data?

Dr Schnickel. The ISHLT [International Society for Heart and Lung Transplantation] working group, as you said, has referenced a PGD grade 3 as less than 200 with chest radiograph infiltrates. When we first wrote this and when we first looked at our data, the ISHLT working group hadn't yet published their abstract, and they just recently presented it as an abstract at the ISHLT. So for that reason and for the reason that our previous work and the work of others has used a ratio of 150 or less, we chose that. In our future work, we definitely agree that we should all standardize our definitions of PGD. We did in fact go back and look at our incidence, and if it's classified as under 200 , the $\mathrm{PaO}_{2} / \mathrm{FIO}_{2}$ ratio, our incidence is $3 \%$ rather than $2 \%$.

Dr Vigneswaran. If your analysis shows such an outcome, it is very good.

Severe primary graft dysfunction is associated with a longer intubation time, length of stay in the ICU, and longer length of hospital stay following lung transplantation. I hope I am not too naive in saying that the reverse should be true; if the primary graft dysfunction is lower, you should have a shorter length of intubation, ICU stay, and hospital length of stay.

Lung transplantation is continuously evolving, and there are always measures taken to improve the technique and conduct of the operation, so any meaningful comparisons can only be made to contemporary reports. The University Healthcare Consortium reporting data during the same years as yours for similar cohorts of patients from participating centers estimated that the hospital length of stay should be about 10 to 11 days following lung transplantation. We reported our experience from the Loyola University Medical Center last year at the ACCP [American College of Chest Physicians] meeting of 117 patients during the same period as yours. The groups are very comparable for etiology, donor-preservation techniques, and types of transplantation. Only $15 \%$ of our patients required $\mathrm{CPB}$, and these were mainly for severe pulmonary hypertension, either primary or secondary. The reperfusion of the allograft was done in the traditional way, by gradually unclamping the pulmonary artery. A critical pathway guided our postoperative management. The early mortality was $5.8 \%$ in our cohort, but the striking difference was that in our cohort, the median length of intubation was 16 hours and the median hospital length of stay was 7 days-almost half what you have reported. Could you comment on the longer intubation time and the prolonged hospital length of stay compared with what was expected in this cohort? Were there any late-onset pulmonary or other medical or nonmedical reasons for this observation?

Dr Schnickel. Loyola is known as an outstanding transplant center in the area of lung transplantation. I applaud your results.

I don't have any explanation for why your intubation time is much better than ours. I mean obviously we did look at days rather than hours, so that may account for some of the difference, but we don't have a protocol per se about the exact time of extubation. I really don't have an explanation why your intubation time is shorter than ours. In terms of the CPB, that's part of our routine procedure for all double-lung transplants. It makes the modified reperfusion easier to instill, and it's just part of our routine. We have found that very effective.

Dr Bryan F. Meyers (St Louis, Mo). You had great clinical results, but I also share concerns that it might not have been achieved by reduction of ischemia-reperfusion injury by your techniques. You reported your $\mathrm{PaO}_{2} / \mathrm{FIO}_{2}$ ratio at 6 hours at 252, plus or minus 120 . Normally you would expect about $60 \%$ of the scores to fall between 1 standard deviation above and 1 below the mean. So I would expect two thirds of your scores to be between 130 and 375 . It seems like there would be room for about $15 \%$ or $18 \%$ of the scores to be below 130. When I look at Dahlberg's presentation in the Journal of Heart and Lung Transplantation in 2004, they reported a median in the 300 to 400 range. When we checked our own results at Washington University for 700 transplants, the mean $\mathrm{PaO}_{2} / \mathrm{FIO}_{2}$ ratio at time 0 was 319 and at time 24 hours was 318 , yet last year we reported an ischemia-reperfusion injury rate of about $22 \%$ using the same threshold as you. I think there is something that doesn't quite add up. It might be your use of the 48-hour time mark, or it might be your inclusion of the radiograph, but it seems like your results are similar to other reported groups, and maybe not even as good if you look at the $\mathrm{PaO}_{2} / \mathrm{FIO}_{2}$ ratio. However, your clinical results are excellent, and I commend you on the report.

Dr Schnickel. Thank you. 